\title{
Editorial
}

\section{Acción Humanitaria: una necesidad en la formación universitaria}

\author{
Consuelo Giménez Pardoㅁ, Manuel Rodríguez Zapata ${ }^{2,}$ * \\ 1 Secretaria Académica de la Facultad de Medicina y Ciencias de la Salud y Directora del Master en Acción \\ Humanitaria Sanitaria; ORCID id: https://orcid.org/0000-0002-8206-1952 \\ 2 Decano de la Facultad de Medicina y Ciencias de la Salud; ORCID id: https://orcid.org/0000-0003-3673-4025 \\ * Autor correspondencia: manuel.rodriguezz@uah.es
}

DOI: https://doi.org/10.37536/RIECS.2020.5.S1.xxx

La Resolución A/RES/46/182 de la 78ª sesión plenaria de Naciones Unidas (NNUU) de 19 de diciembre de 1991 indica que ayuda humanitaria es todo lo que comprende las condiciones recogidas en dicha resolución y que solo puede ser referido a ellas.

Se guía por principios como la Independencia, que implica autonomía frente a los objetivos políticos, económicos, o militares; Neutralidad, que significa no tomar partido en las hostilidades de orden político, religioso, racial e ideológico e Imparcialidad, que conlleva que las acciones humanitarias se lleven a cabo a partir de las necesidades de la población sin distinción sobre raza, sexo, religión, clase u opinión política. Otro principio, no recogido en la resolución, es el de Humanidad que implica proteger la vida y garantizar el respeto a los seres humanos, quizá el principio de mayor importancia al poner a los seres humanos en el centro de las respuestas.

La ayuda humanitaria requiere de evaluación previa de la situación por organismos independientes y pretende salvar vidas, curar enfermedades y aliviar el dolor de las víctimas. Los mecanismos para poner en marcha una ayuda humanitaria ocurren cuando el gobierno legítimo entiende que la situación supera sus capacidades y solicita ayuda internacional; en el caso de que no haya gobierno esta ayuda humanitaria la impulsan los organismos de NNUU que tienen competencias en este tema.

Según el informe La acción humanitaria en 2018-2019: sin cambios en tiempos de incertidumbre, realizado por Médicos sin Fronteras (MSF) y el Instituto de Estudios sobre Conflictos y Acción Humanitaria (IECAH), a partir del último ejercicio del que se tienen datos (2018) se ha producido un leve incremento del presupuesto para acción humanitaria por la Agencia Española de Cooperación Internacional para el Desarrollo (AECID) con una concentración de la ayuda de emergencias para ayuda y rehabilitación y prevención de desastres que no supone ni el 1\% del total de los fondos. Los recursos para hacer frente son escasos, pero se observan avances como la aprobación reciente de una Estrategia de Acción Humanitaria de la Cooperación Española 2019-2026 o que por primera vez se movilizan los equipos START (Spanish Technical Aid Response Team) y la coordinación para promover acciones conjuntas en materia humanitaria a través del convenio firmado por la AECID, Comunidades Autónomas (CCAA) y la Federación Española de Municipios y Provincias (FEMP).

La existencia de países vulnerables con malas condiciones de salubridad, recursos escasos y sistemas sanitarios deficientes junto con un panorama mundial geopolítico y socio-económico cada vez más complejo, supone un reto enorme que exige a las agencias internacionales cumplir con requerimientos de calidad cada vez mayores. Entre otras muchas cuestiones es preciso que el personal que trabaja en este campo reciba la mejor formación. No es la primera vez que en la revista RIECS se tratan temas relacionados con diversos aspectos de la acción humanitaria (RIECS, 2017, 2, 1; RIECS, 2017, 2,2).

La implicación de las universidades en la realidad social en la que se desenvuelven y el compromiso con esa realidad, implica la creación de programas formativos teórico-prácticos, que no existen de modo específico en las universidades, relacionados con los problemas de salud global, movimientos migratorios, historia mundial, geopolítica, los derechos, antropología, la seguridad, la 
atención sanitaria a poblaciones vulnerables, los planes sanitarios concretos de actuación, la intervención en proyectos...etc. Así, se hace necesario dotar de herramientas a los estudiantes de aquellas cuestiones que no se explican en los estudios de Grado de las profesiones sanitarias.

La Facultad de Medicina y Ciencias de la Salud de la Universidad de Alcalá manifestó en el curso académico 2015-2016 su compromiso en este tipo de formación y así, atendiendo a las demandas sociales globales, se gestó un proyecto de Posgrado entre la Facultad y la organización de acción humanitaria Médicos del Mundo que comenzó su andadura en el curso 2017-2018, que actualmente se encuentra en su tercera edición y que recientemente ha recibido el Primer Premio de la Fundación de Educación Médica 2019, Lilly-UCM a la mejor formación en enseñanza de posgrado.

Así, el Master en Acción Humanitaria Sanitaria (MUAHS), es un master oficial semipresencial de 90 ECTS, de año y medio de duración. Por delante, 2.160 horas de formación divididas en diez módulos con prácticas modulares presenciales y prácticas en terreno en diversos países y en el que participan entidades como Cruz Roja (marco legal, DIH), Oxfam (agua y saneamiento), Medicus Mundi (salud pública y salud global), MSF (atención sanitaria en zonas de conflicto), Amref Salud África (salud y sistemas sanitarios en África), Farmamundi (logística de fármacos, seguridad y transporte inmediato de medicamentos). https://master-universitario-en-accion-humanitaria-sanitaria-muahs8.webnode.es/

En este sentido la Facultad de Medicina y Ciencias de la Salud considera su implicación en la responsabilidad social ante un mundo cambiante y con este posgrado oficial se convierte en la única Facultad de Medicina de una universidad pública que oferta como centro un estudio de este tipo orientado a la formación de profesionales de la acción humanitaria sanitaria. Todo un reto docente y logístico que mueve cerca de 70 profesores y profesionales de diversas disciplinas por el que esta Facultad sigue apostando.

(C) 2020 por los autores; Esta obra está sujeta a la licencia de Reconocimiento 4.0 Internacional de Creative Commons. Para ver una copia de esta licencia, visite http://creativecommons.org/licenses/by-nc-nd/4.0/. 